María Ximena Álvarez

\title{
Repensando identidades en el exilio en Sudamérica: artistas judeo-alemanes y su lucha en el campo de la cultura (1938-1953)
}

Con el ascenso del nacionalsocialismo y la progresiva extensión de leyes antisemitas, se estima que medio millón de personas quedaron excluidas de su comunidad, de su sociedad y fueron hechas extranjeras en su propia patria: los alemanes de ascendencia judía. Aquellos que por medio de la huida del Tercer Reich salvarán sus vidas, se enfrentarán en el exilio a una dura reflexión identitaria: ¿Cuál es en definitiva su patria y su lugar de pertenencia? ¿Qué lugar ocupaba Alemania, y qué Alemania, en su identificación? ¿La huida significaría una ruptura total con su pasado europeo?, ¿asimilación a la sociedad de acogida?, ¿regreso a las raíces judías? ¿Sería posible defender la existencia de una "otra Alemania" que no fuera la de Hitler?

Estas reflexiones identitarias deseo realizarlas mediante el análisis de tres ejemplos de artistas de habla alemana que huyeron hacia América del Sur, quienes se convirtieron en figuras destacadas en las sociedades de emigrados. Sin representar los grandes nombres de las artes y las letras alemanas, sino el común denominador de los emigrantes, son tres casos exitosos, por haber logrado trabajar y actuar en su propio idioma, en un ambiente lingüístico y cultural extraño. Hicieron de su "Babilonia", de su diáspora un espacio de creatividad, transformando el duro período del exilio en uno de provechosa producción.

Como le expresara Hannah Arendt a Karl Jaspers: "Para mí Alemania es la lengua materna, la filosofía y la poesía". "Al igual que Arendt, para estos artistas, como tantos otros emigrados, su lengua materna se convirtió en su lugar de pertenencia, así como también la herramienta de su arte.

1 En lo que sigue, los textos aquí citados en inglés o alemán son de traducción libre de la autora al castellano. Las citas originales se ponen en notas a pie de página. Arendt (2007: 205): "Für mich ist Deutschland die Mutttersprache, die Philosophie und die Dichtung". 
El actor, escritor y director teatral alemán Paul Walter Jacob se afincará en Buenos Aires en enero de 1939, fundando junto a su esposa, la actriz Liselott Reger, y el con el gran apoyo de círculos de emigrados, en especial del director del diario Argentinisches Tageblatt, el Dr. Ernesto Alemann, la companía teatral Freie Deutsche Bühne (FDB) -Teatro Alemán Independiente- en abril de 1940.

En el Uruguay, el abogado originario de Fráncfort del Óder, Hermann P. Gebhardt, funda en agosto de 1938 una radioemisora de habla alemana, Die Stimme des Tages -La voz del día (LVD)-, la única radioemisora germano-española democrática de la costa Atlántica, un foro de voces alemanas antifascistas en el Uruguay.

En Bolivia, por su parte, el abogado, escritor y actor austríaco Fritz Kalmar se integrará activamente a la vida cultural creada por los emigrados en La Paz. En 1941 co-funda y luego preside la Federación de Austríacos Libres (FAL), centro de la lucha antifascista y también cultural de la emigración de habla alemana en Bolivia.

Las investigaciones del exilio utilizan el término exiliado para referirse a aquellos que por motivos políticos huyeron de la Alemania nazi. Este grupo, que constituye una minoría en la gran masa de la emigración (30 mil aproximadamente), vivió una primera etapa de su exilio en suelo europeo, deambulando por varios países, hasta que la extensión del dominio nazi con el inicio de la guerra hizo ya imposible la permanencia en dicho continente. Entendían la salida de Alemania como una situación transitoria, y su regreso se realizaría tan pronto las condiciones políticas lo permitieran, o sea, tras el derrocamiento de Hitler. Frente a este minoritario grupo de exiliados se encuentran los emigrantes (aprox. 500 mil), de los cuales el 90\% eran judíos o los considerados Mischlinge, tras las leyes raciales de Núremberg de 1935. Esta masa migratoria se produjo tras los excesos de violencia de la Kristallnacht en noviembre de 1938, y, a diferencia de los primeros, entendían su partida como una situación definitiva, una ruptura total con su pasado europeo, un irse para no volver (Krohn/Zur Mühlen/ Paul/Winckler 1998). A los efectos de este trabajo, se utilizará indistintamente el término exilio o emigración, ya que se entiende la diferencia por el estado transitorio en el primer caso, y, definitivo, en el segundo. No es posible saber, quizás tampoco lo supieran ellos en el período de sus primeros años en América Latina, cuál sería la decisión a tomar, transformar su salida de Europa en una situación transitoria, o 
sea, de exilio, o permanente, de emigración. Esta disyuntiva, "exilio o migración", atiende a muchos, y constituye una temática compleja en las sociedades de "emigrados". Algunos "exiliados" se convierten en migrantes, y otros, quienes se habían planteado nunca más volver, regresan, ya que otras condicionantes (familiares, económicas, etc.) influyen en dicha decisión.

\section{Alemanes en una América latina}

América Latina, un subcontinente hasta ese momento desconocido y exótico, alejado no sólo geográfica, sino culturalmente de los países de lengua germana, no fue por lo general el primer destino elegido, sino la "alternativa posible", así el título de un simposio organizado por Karl Kohut y Patrik von zur Mühlen (1994). La relativa facilidad para adquirir visas para países como Bolivia, Ecuador, Uruguay llevaron a que miles de personas que apenas poseían mínimos conocimientos geográficos sobre los mismos, los convirtieran en sus nuevos lugares de residencia, ya que importaba simplemente huir. La dura vida como emigrantes en sociedades con un idioma y una cultura ajenos, generó densas redes de solidaridad, comunicación e intercambio entre los emigrados de lengua alemana que traspasaban las fronteras nacionales de los países de acogida. Fue así como medios de prensa, compañías teatrales, actividades políticas circulaban en Sudamérica, convirtiéndose en elementos vinculantes de las sociedades de emigrados, muchas veces más cercanas a sus connacionales alemanes en México o Bolivia que a su vecino uruguayo:

El sentimiento de malestar o bien de sentirse diferentes en el país de la diáspora, lleva a la identificación con otras comunidades co-étnicas en otros países. Vínculos como lenguaje, religión y cultura y el sentirse parte de un destino en común impregnan este tipo de relaciones transnacionales y les dan una cualidad afectiva e íntima $[\ldots]^{2}$

Estos tres personajes, Paul Walter Jacob, Fritz Kalmar y Hermann P. Gebhardt, separados por grandes distancias geográficas, consiguen traspasar las fronteras de los países que les dieron acogida, conocién-

2 Cohen (1997: 124): "The sense of unease or difference that diaspora peoples feel in their country of settlement is paralleled by a tendency to identify instead with coethnic communities in other countries. Bonds of language, religion, culture, and a sense of a common fate impregnate such a transnational relationship and give to it an affective, intimate quality [...]". 
dose y trabajando juntos en el exilio. Comparten un pasado de persecución por sus raíces judías, una misma convicción política junto a la socialdemocracia y, sobre todo, su amor por la lengua y la cultura alemana, el lazo profesional que los unirá en esos años. La compañía teatral de Jacob realizará temporadas en Montevideo, organizadas por la radio de Gebhardt, Die Stimme des Tages (LVD). Jacob será también frecuente locutor en dicha radio, aún luego de su regreso a Alemania. Kalmar y su esposa, la actriz Erna Terrel, estarán en contacto con la compañía teatral de Jacob, en la cual Terrel actuará como invitada, así como en la radio de Montevideo. Estos vínculos profesionales con LVD y con la comunidad de germano parlantes asentados en Montevideo, serán decisivos para que Kalmar y Terrel se trasladen al Uruguay en la década de los cincuenta, luego de más de un decenio de residencia en Bolivia.

Los tres consiguieron convertir las condiciones desventajosas del exilio en elementos claves de su éxito profesional: las dificultades económicas e impedimentos legales, como a su vez el problema de la diferencia lingüística y cultural.

La imposibilidad para los abogados Gebhardt y Kalmar de ejercer su profesión en Uruguay y Bolivia, los llevó a concretar antiguas inquietudes, en el periodismo, el primero, y en el teatro, el segundo. Para Jacob, que ya traía una experiencia de actuación teatral en Europa, los diez años en que vivió y dirigió su teatro en Buenos Aires fueron los más fructíferos de su carrera, período que no se volvería a repetir a su regreso a Alemania. Fue la experiencia profesional en Buenos Aires, su carta de presentación en la inserción laboral de la posguerra. El exilio, en la experiencia de Jacob, no fue una "Sala de Espera" improductiva, Wartesaal, al decir de Lion Feuchtwanger, entre la partida y el regreso a Alemania.

Jacob, Gebhardt y Kalmar no se conformaron con actuar en el ámbito de la cultura, sino que en dicho marco se comprometieron en las actividades de los alemanes antifascistas en Sudamérica. Dicho compromiso político en la defensa de una Alemania diferente del nacionalsocialismo debió sortear variadas dificultades, que se intensificaban dependiendo de la situación política de los países de acogida. Frente a estos grupos de emigrados que huían del nazismo -por razones políticas, por su ascendencia judía, o bien por ambas-, se situaba la colonia de alemanes residentes en el país, quienes se adhirieron en su gran 
mayoría a los valores propagados por el Tercer Reich. La "quinta columna", o bien, como les llamaban jocosamente en Argentina, los Nazioten-Nazi-Idioten- (Naumann 1985: 98), constituían una latente amenaza frente a las actividades de los alemanes democráticos, convirtiéndose en algunos casos en real: boicot de la exhibición de la obra de Ferdinand Bruckner, Las Razas, en 1934 y, en el mismo año, un atentado contra el diario alemán democrático Argentinisches Tageblatt, son algunos ejemplos. Del mismo modo, el peligro de que los gobiernos de residencia, en el convulsivo contexto de la guerra mundial, frenaran sus actividades o, en el caso extremo, los expulsaran del país, era en el caso de Argentina un temor más que fundado. Otros escollos que debieron sortear para el fluido desarrollo de sus actividades políticas provenían desde dentro del círculo de los emigrados y fue un común denominador en los diferentes países de la región: la censura absoluta por parte de las instituciones judías de todos aquellos judeo-alemanes integrados en la lucha antifascista, y, por último, la rivalidad insoslayable entre comunistas y socialistas que impidió la real concreción de un frente común.

\section{Paul Walter Jacob en Argentina}

El exilio en Buenos Aires de Paul Walter Jacob (Duisburg, 1905 Schwäbisch Hall, 1977) fue una excepción, contando con ciertas ventajas de las cuales no muchos podrían jactarse. Viajó legalmente y en primera clase acompañado de la actriz Liselott Reger, ciudadana argentina, quien se convertiría en su esposa a pocos meses de arribar a suelo sudamericano. Se afincó en una metrópoli rica que contaba con 2 millones y medio de habitantes, de los cuales 100 mil hablaban alemán. Ahora se abría el epicentro cultural del subcontinente sudamericano, la spanisches New York, como él (Jacob) la llamaba (Naumann 1985: 83), la colonia más grande de emigrados de habla alemana en Latinoamérica. En abril de 1940 realiza su primera presentación la compañía que fundó: Freie Deutsche Bühne -Teatro Alemán Independiente.

Como su director y actor, logró convertirla en un centro cultural importante de la colonia de alemanes exiliados en Buenos Aires, y también fuera de las fronteras argentinas, realizando temporadas en Uruguay. No se limitó su actuación al teatro, sino que escribió en va- 
rios medios de prensa alemanes y argentinos, fue crítico musical, conferencista y escritor.

$\mathrm{Su}$ actividad política en este contexto no es fácil de definir. Por un lado, fue cara visible en diversas actividades antifascistas. Por otro, su teatro y las obras allí exhibidas, en su mayoría comedias, decepcionan cualquier posibilidad de ver su escenario como un "teatro combativo", el espacio en que se desarrolló su compromiso político.

A su llegada a Argentina se vincula rápidamente con las organizaciones sociales y políticas antifascistas, como el diario Argentinisches Tageblatt (fundado en 1882), la asociación socialista Vorwärts (1882), Das Andere Deutschland (DAD) -La otra Alemania- (organización socialista fundada en 1937), así como el medio de prensa comunista Volksblatt (1941-1943). Se convierte en un asiduo conferencista sobre temas musicales y de teatro alemán en ambas capitales del Plata, y se destaca especialmente su participación como uno de los oradores del Congreso Sudamericano de Antifascistas, organizado por el movimiento socialista DAD en Montevideo en enero 1943.

Sin embargo, la posibilidad de luchar con el espíritu de su teatro debió enfrentar a dos grandes enemigos: una Argentina "neutralmente profascista" y un público mayormente despolitizado.

El temor de censura de las piezas por parte del gobierno, o bien el "boicot" de la numerosa comunidad alemana nacionalista se hace visible en sus cartas. Por este motivo, la obra antifascista Watch on the Rhine de Lillian Hellmans, presentada en agosto de 1942, es traducida por su esposa Liselott Reger-Jacob como Die Unbesiegten -Los invictos- para no llamar la atención sobre el contenido político de la misma. Asimismo, Jacob le escribe al crítico teatral del Argentinisches Tageblatt, el Dr. Pauly, con la solicitud de evitar todo comentario sobre su contenido antes de ser exhibida, sino "deberemos contar con intentos de boicot y con dificultades administrativas". ${ }^{3}$ Luego de la presentación no habría problema, muy por el contrario, citar el nombre original de la pieza sería muy provechoso para la futura presentación que se planeaba en Montevideo, "allí se hará con el título original y con gran propaganda sobre su contenido (ya que esto no sólo es posi-

3 Para esta cita y la siguiente ver nota 4. 
ble sino esperado)". ${ }^{4}$ Con ello se destacan las diferencias entre las libertades que gozaban los antifascistas de una y otra orilla. Sin embargo, la despolitización del público era una constante en ambos márgenes del Plata. Un espectador le escribe a Jacob luego de que la FDB se presentara en Montevideo con el drama Hombres a la deriva de Vilém Werner:

Comprendo ciertamente que con la exhibición de piezas de este carácter debe contar con bancos vacíos, ya que las personas en la lucha por la existencia en un medio desconocido, no tienen ni el carácter ni los nervios para ver este tipo de obras.

-lo incita a realizar nuevas actividades políticas con su teatro-: "No sería quizás visitado por un público masivo, pero sí por un público de calidad" (mis subrayados, M. X. A.). ${ }^{5}$

El lugar central que ocuparon las comedias y las operetas frente a los dramas de contenido político, dan a entender que el público masivo fue preferido al de calidad (Trapp 1991; Pohle 1987a; 1987b; 1988). ${ }^{6}$

A su vez, su vínculo a DAD, y ser imagen visible de la lucha antifascista le costó la antipatía de las organizaciones judías, quienes censuraron, boicotearon y criticaron duramente a aquellos judíos relacio-

4 Carta de Paul Walter Jacob al Dr. Pauly, 27 de agosto de 1942. Walter-A.-Berendsohn-Forschungsstelle für deutsche Exilliteratur (BFfdE), Archivo Paul Walter Jacob (PWJ), correspondencia 1942. "Ich muss leider annehmen, dass, falls das andere Lager durch die Kritik auf Inhalt und Tendenz aufmerksam gemacht wird, wir mit Störungsversuchen und event. behördlichen Unannehmlichkeiten rechnen müssten. Dagegen wäre ich Ihnen für eine besonders eingehende Besprechung unter Angabe des Originaltitels und der Tendenz am Montag zu besonderem Danke verpflichtet, da wir das Stück im November in Montevideo geben wollten, und wir dort gerade mit Originaltitel und -tendenz gross werben wollen (was dort ja möglich und erwünscht ist)".

5 Carta de Fred Stoessel a Paul Walter Jacob, 12 de diciembre de 1940. BFfdE, PWJ, correspondencia 1940. "Ich sehe natürlich voll ein, daß Sie mit Stücken dieses Charakters bald vor leeren Bänken spielen würden, da die Menschen durch die Schwere ihres Existenzkampfes in der Fremde nicht die Gefühle u. die Nerven haben, solches zu sehen. [...] Das wären viell. keine Veranstaltungen mit Publikumsmassen, dafür aber mit Publikumsqualitäten”.

6 Tanto Trapp como Pohle destacan la falta de compromiso político del teatro de Jacob. El título de Trapp (1991), por ejemplo, es muy elocuente al respecto: "Zwischen Unterhaltungsfunktion und der Erwartung politischer Stellungnahme". 
nados con la defensa de una Alemania diferente al nazismo, tildándolos de traidores.

El recelo y desconfianza que éstas le tenían a Jacob se mantuvo por varios años, como se desprende de la correspondencia entre éste y organizaciones como el Jüdische Wochenschau -JW-, Semanario Israelita (fundado en 1940 por el sionista Hardi Swarsensky y Günter Friedländer). En una carta de Jacob dirigida al presidente del JW, Friedländer, en agosto de 1944, solicita apoyo para su teatro, utilizando como argumentación la composición mayormente judía del mismo. Le reprocha haber organizado un costoso concierto con la orquesta del Teatro Colón "que ni fomenta la cultura judía [...] ni está integrada por judíos $[\ldots] ” .7$

En este caso, el judaísmo es apelado en interés promocional de su teatro. En su identidad pesaba más su integración a la nación alemana, definiéndose como un hombre enraizado en la cultura y el idioma alemán. En sus conferencias mencionaba a "los artistas judíos expulsados de Europa" como si él no formara parte de ellos, causando cierta risa entre sus espectadores. ${ }^{8}$

Jacob, a pesar de ser un alemán poco identificado con su judaísmo, nunca dejó de vincularse a los grupos judíos, quienes constituían el público de su teatro. Entendía la partida de Alemania, como la gran mayoría de exiliados políticos, como una situación transitoria y no definitiva. Cuando en su remigración en Alemania le preguntaban por qué había vuelto en un momento en que muchos querían salir de las duras condiciones vividas en la posguerra, era muy elocuente su respuesta: "No deseaba otra cosa, que volver a trabajar en mi idioma. Yo

7 Carta de Paul Walter Jacob a Günter Friedländer, 7 agosto de 1944. BFfdE, PWJ, correspondencia 1944. "Man hat im vorigen Jahre ein sehr teures Konzert mit dem gewiss nicht unterstützung-bedürftigen Colón-Orchester veranstaltet [...], aber man hat es systematisch unterlassen, mit der "billigen" FDB zusammenzuarbeiten. Irgendein 'jüdischer Standpunkt' kann hier nicht als Begründung herangezogen werden, denn weder bot das Colón-Orchester [...] irgendeine Möglichkeit jüdisch betonter kultureller Arbeit [...], noch gehören diesem Orchester jüdische Emigranten an. Die FDB besteht, wie Sie wissen [...] zu 90\% aus jüdischen Emigranten [...]".

8 Entrevista de Uwe Naumann a Helmuth Freund, 2 de julio de 1984. BFfdE, PWJ, VII c) 408 . 
soy un alemán, [...] y por tanto, sólo puedo vivir en un espacio lingüístico alemán". 9

\section{Hermann P. Gebhardt en Uruguay}

Al otro lado del Río de la Plata, en la capital montevideana, se encuentra desde 1937 el abogado Hermann P. Gebhardt (Fráncfort del Óder, 1903 - Montevideo, 1984). En agosto de 1938 sale al aire la radioemisora de habla alemana democrática La voz del Día-Die Stimme des Tages- (LVD), que, como señalara su fundador: a pesar de que sus amigos más optimistas no le daban más de seis meses de vida, precedió a su creador, quien falleciera en 1984. Que el objetivo de Gebhardt al fundar la radio era político, lo expresó él mismo en varias oportunidades. Si bien la necesidad de ganarse el sustento en una tierra cuyo idioma no dominaba estaba presente, lo motivaba también una deuda ética por sentir haber hecho muy poco contra el nacionalsocialismo en su tierra natal. Los minutos más esperados eran la síntesis diaria de la política internacional que el Dr. Gebhardt realizaba bajo el título Die Welt von Heute -El mundo de hoy-. Allí, en breves minutos realizaba una puesta a punto de los aconteceres políticos con una visión crítica, haciendo de ésta su característica fundamental, ganando un respetado lugar en la comunidad de emigrados. También ofrecía en ella espacio para asociaciones políticas y religiosas, aún cuando no comulgaba con sus ideas, como los minutos que disponían los sionistas los lunes, o bien los jueves, los comunistas del Comité Alemán Antifascista. El respeto que éste mostraba hacia todas las tendencias antifascistas se denota en el programa especial de celebración de la caída de Berlín el 2 de mayo de 1945, cuando junto a la Marsellesa se pasa el himno nacional ruso. Es digno de destacar que el diario uruguayo de tradición batallista El Día se negó a izar la bandera soviética junto a la de los otros aliados al finalizar la guerra, dando con ello muestra del convencimiento democrático que promulgaba Gebhardt.

Sin embargo, el compromiso político asumido por Gebhardt, al igual que le sucedió a Jacob en Argentina, sufrió ciertos desencuentros con la institución que unía a los alemanes judíos en el Uruguay, la

9 Jacob en Naumann (1985: 11): "Ich habe mir nichts sehnlicher gewünscht, als wieder in meiner Sprache zu arbeiten. Ich bin ein Deutscher [...] und ich kann nur im deutschen Sprachraum wirklich leben”. 
Nueva Congregación Israelita -NCI- (fundada en junio de 1936), como lo ocurrido entre noviembre y diciembre de 1942. En esa época emerge un conflicto tras una conferencia organizada en noviembre de 1942 por el Comité Alemán Antifascista en el Ateneo de Montevideo, bajo el lema "Alemania no es Hitler". En dicha oportunidad, Dr. Gebhardt, junto a otras personalidades democráticas uruguayas, pronuncia un discurso defendiendo la existencia de una Alemania diferente del nazismo, como alegaba el título del evento. Un representante de la Nueva Congregación Israelita, el Dr. Karl Berets, le increpa en una carta abierta dicha participación. Recibiendo el apoyo del resto de los representantes de la NCI, le expresa su desacuerdo, considerándolo un traidor a la comunidad judía que él integra. La postura de Berets no sólo es defendida por la NCI de Montevideo, sino que dicha carta se publica en el Jüdische Wochenschau de Buenos Aires.

Su radio se autodenomina democrática y alemana. ¿Por qué mi estimado Dr. Gebhardt entre sus trabajadores se encuentran en su mayoría sólo judíos, y algunos uruguayos, los clientes que financian su radio son judíos, y algunos uruguayos? ¿por qué existen sólo judíos "Amigos de la Voz del Día" con la excepción de algunos pocos uruguayos? ¿Dónde queda su infeliz y unilateral amor por los alemanes que no son judíos? La respuesta: [Alemania] es Hitler. ${ }^{10}$

Gebhardt envía una larga carta a la NCI en respuesta. Entre los varios argumentos expresados, aclara su sorpresa y pregunta por qué recibe él dichas críticas y no, por ejemplo, las potencias aliadas, la BBC de Londres, los artistas e intelectuales alemanes -también judíos- propuestos a luchar contra el nazismo y no contra la nación alemana. Destaca Gebhardt:

El asunto sobrepasa hacia una pregunta interna judía. El futuro del mundo es la pregunta clave para todos lo hombres libres, independiente de la raza o la religión a la que pertenezcan. Yo no entro en su mundo privado,

10 Carta abierta del Dr. Karl Berets a Hermann P. Gebhardt. Montevideo, 29 de noviembre de 42, PWJ, VII c) 408: "Ihre Rundfunkstunde nennt sich eine demokratische deutsche. Warum, geehrter Herr Dr. Gebhardt, sind Ihre zahlreichen Mitarbeiter fast ausschliesslich Juden - \& vereinzelt - Uruguayer, warum sind Ihre Kunden, die die Rundfunkstunde finanziell erhalten, fast ausschliesslich Juden $-\&$ vereinzelt Uruguayer -, \& warum gibt es nur jüdische "Amigos de la Voz del Día" ausser vielleicht wenigen Uruguayern? Wo bleiben m.a.W. die Objecte Ihrer unglücklichen \& einseitigen Liebe, die Deutschen d.h die nichtjüdischen Deutschen? -Antwort: Es Hitler". Aquí, Berets juega con el título de la conferencia que en español era "Alemania no es Hitler". 
si su patria espiritual del mañana será Palestina. Yo no me dejo expulsar de Occidente y por ello lucharé con mis débiles fuerzas para pensar en conjunto el futuro de Europa junto a todos los antifascistas y enemigos del fascismo [...]. Yo lucho junto con los antifascistas cualquiera sea su raza, nacionalidad, religión contra los fascistas de cualquier raza, nacionalidad y religión [...]. ${ }^{1}$

La respuesta de Gebhardt no es publicada en el Boletín Informativo de la NCI, tampoco en el JW. ${ }^{12}$ Estas diferencias con la NCI no derivarán en mayores conflictos, ya que la radio de Gebhardt será luego de la guerra el puente entre la comunidad judía alemana y la República Federal, siendo su propósito el aunar a judíos y alemanes. En 1950, con la llegada al Uruguay del primer embajador de la República Federal, el socialdemócrata Gustav Herbig, utilizará la radio para dirigirse a la comunidad judía alemana, afirmando así mejores relaciones entre éstos y el gobierno de Bonn.

En una comunidad de emigrados tan pequeña como la formada en el Uruguay, jugó la radio un papel esencial, como informativo, centro cultural y, sobre todo, de cohesión. El escuchar "lo que el Dr. Gebhardt dijo" se convirtió en una actividad del día a día de los alemanes democráticos del Uruguay. ${ }^{13}$ En un primer momento en que el idioma no se manejaba, era su contacto con el mundo exterior, y el puente cultural hacia la patria perdida. Los "años de gloria" de la radio fueron también los más difíciles de la emigración, aquellos en que el nazismo aún regía en Europa.

Si bien no se identificaba religiosamente con el judaísmo, existía en Gebhardt un compromiso moral hacia el grupo al cual se sentía pertenecer. "Sólo las ratas abandonan el barco cuando se hunde", era

11 Carta abierta de Hermann P. Gebhardt a Karl Berets. 14 de diciembre de 1942, PWJ, VII c) 408: "Diese Angelegenheit übersteigt bei weitem den Rahmen einer internen jüdischen Frage. Die Zukunft der Welt ist die Kernfrage für alle freiheitliebenden Menschen, gleichgültig welcher Rasse oder Religion sie angehören. Ich rede Ihnen nicht in Ihre Privatentschlüsse herein, wenn Sie Ihre geistige Heimat von morgen nach Palestina verlegt haben. Ich lasse mich nicht aus dem Abendlande vertreiben \& werde mit meinen schwachen Kräften an der Zukunft Europas mitdenken, gemeinschaftlich, mit allen Antifaschisten \& in Gegnerschaft zu allen Faschisten. [...] Ich kämpfe mit allen Antifaschisten jeder Rasse, jeder Nation \& jeder Religion gegen die Faschisten jeder Rasse, jeder Nation \& jeder Religion [....]".

12 Carta de Hermann P. Gebhardt a Paul Walter Jacob. 7 de enero de 1943, BFfdE, PWJ, correspondencia 1943.

13 La Plata Post, 27 de mayo de 1968. 
una frase que se le oía decir, al referirse a su condición judía. ${ }^{14}$ Por su parte, el papel de mediador a través de su radio para hermandar a los judíos con Alemania no es por todos aceptado, recibiendo críticas de ambos lados. "Por eso los judíos extremistas dicen que soy demasiado alemán, y los alemanes extremistas que soy demasiado judío". ${ }^{15}$

Su integración al Uruguay se produjo naturalmente, aprendió fácilmente el español y expresó ya no poder imaginar otro lugar donde vivir. Valoraba las libertades de la democracia uruguaya, ya que se le permitió continuar ininterrumpidamente con la transmisión de su radio aún luego de que el Uruguay en enero de 1942 rompiera relaciones con las potencias del Eje. A su vez, se vinculó rápidamente con la intelectualidad democrática y con políticos liberales del país. El futuro presidente, el político colorado Luis Batlle Berres, como el diputado socialista, el Dr. Cardozo, eran figuras presentes en su radio. Luego de la guerra, sin plantearse la idea de volver, se mantiene unido a su antigua patria a través de sus actividades periodísticas como corresponsal de prensa de medios alemanes, y por su cercano contacto con la embajada de la Alemania Federal en Montevideo.

\section{Fritz Kalmar en Bolivia}

La odisea que el austríaco Fritz Kalmar (Viena, 1911 - Montevideo, 2008) vive antes de iniciar su exilio boliviano, da muestra del destino que siguieron miles de emigrados, obligados a salvar las dificultades burocráticas para abandonar Europa. La masa de la emigración austríaca se produjo entre el Anschluss, en marzo de 1938, y la Kristallnacht en noviembre del mismo año. Consiguieron huir de Austria entre 1938 y 1941 más de 125 mil personas, siendo el 90\% de éstos judíos. Tan sólo 12 mil tenían como destino América Latina (Kloyber 2002: 33). Si bien en Alemania la política antisemita se fue dando progresivamente, el antisemitismo nazi se implantó en Austria, el nuevo Ostreich alemán, en unos meses, casi de la noche a la mañana. Luego de la llamada "Noche de los Cristales Rotos" -9 de noviembre de 1938-, Kalmar consigue huir embarcándose en un navío pesquero

14 Entrevista de la autora a Irene Gebhardt, Freudenheim, junio de 2009.

15 "Interview mit Dr. Gebhardt". En: La Plata Post, 27 de mayo de 1968, PWJ, VII c) 408, p. 4: "So sagen die extremen Juden, ich sei zu deutsch, und die extremen Deutschen, ich sei zu jüdisch". 
noruego a Inglaterra, y arriba medio año después a Perú, para encaminarse finalmente en 1940 a La Paz. Bolivia, uno de los países más pobres del subcontinente latinoamericano, no ofrecía condiciones económicas para que este doctor en Leyes egresado de la Universidad de Viena tuviera más opciones que la de trabajar como empleado en una fábrica de instalaciones eléctricas, como pintor de casas, y luego como profesor particular de inglés, en la dura lucha por el sustento.

Este fue el común denominador de la emigración: la pérdida de estatus y nivel económico. Aquellos que se dedicaron a las actividades artísticas con exclusividad, como Jacob y Gebhardt, constituyen mínimas excepciones; por lo general tenían otra actividad laboral que les proporcionaba el sustento, muy inferior a aquélla que disfrutaban antes de la emigración, por lo menos en los difíciles primeros años.

En ese contexto desfavorable para el surgimiento de una fructífera actividad cultural y política, Fritz Kalmar funda junto con otros austríacos emigrados en 1941 la Federación de Austríacos Libres, integrada desde 1943 al Free Austrian Movement -con sede en Londres-, centro de su actividad política y cultural.

El exilio y la oposición política de los emigrados austríacos como Fritz Kalmar difería del de sus colegas alemanes, el caso de Jacob y Gebhardt, a pesar de haberse integrado en el conjunto de las actividades antifascistas. Habían huido de un país que no existía más, transformándose a partir de marzo de 1938 en el Ostmark del Tercer Reich -frontera oriental del Reich. Su función política y cultural no sería ya diferenciar solamente su patria de los valores del nazismo, como los alemanes, sino reivindicar su existencia. Pero, por otra parte, al poder declararse como "austríacos", el gobierno no tomó medidas contra ellos, como sí lo hizo contra los alemanes, luego de que Bolivia rompiera relaciones con el Reich en 1942 (Blaschitz 1995: 75).

Para el escritor Alfredo Bauer, la gran diferencia entre los emigrados judíos alemanes y austríacos radicaba en su compromiso político, más importante en el caso de estos últimos (Bauer 1995: 113). De los 225.000 judíos que vivían en la década de los 20 en Austria, un $80 \%$ de ellos lo hacían en su capital, la llamada en la época "Viena roja", fuertemente impregnada por la socialdemocracia (Spitzer 2003: 54), como el caso de Fritz Kalmar y su familia.

La Federación de Austríacos Libres, presidida por Kalmar, reunía en sí diferentes tendencias políticas antinazis, promoviendo la lucha 
por la independencia de una Austria libre de fascismos, motivo por el cual consiguió aunar en su seno a círculos burgueses, al lado de comunistas y socialistas (Spitzer 2003: 116-117). Los austríacos en Bolivia, de diferentes tendencias políticas, se integraron casi sin excepciones en la Federación, al contrario de los alemanes, para quienes fue muy difícil establecer un frente común, como lo denotan los enfrentamientos en Argentina y Uruguay.

Las actividades de la Federación variaban entre conferencias sobre temas políticos de la actualidad, exhibición de películas, presentación de piezas de teatro, conciertos en los que se recaudaban fondos para ayudar a los aliados en la guerra contra Hitler. A partir de 1944, con la nueva sede propia que disponía de un escenario, la presentación de obras pudo ser más frecuente. La Federación no sólo constituía una agrupación política, sino que cumplía una función muy importante en la integración de los exiliados, un respiro para el alma, para curar la nostalgia.

Con respecto a los vínculos de Kalmar entre su antigua patria y la patria del exilio, su leve identificación y asimilación a la vida boliviana, es característica de la casi totalidad de emigrados en dicho país. No fue visto como una patria definitiva, sino, al decir de Leo Spitzer (Spitzer 2003), como un Hotel Bolivia, la primera etapa de una nueva emigración hacia EE.UU., Israel u otros países latinoamericanos. Así, luego de 14 años de exilio boliviano, se dirige al Uruguay, en donde se asentará a partir de 1953. Siempre mantuvo un profundo amor a su Austria natal -trabajó para recaudar fondos para la reconstrucción luego de la guerra-, y asumió el cargo de cónsul honorario de Austria en el Uruguay hasta 1992.

El compromiso político y cultural de Kalmar no finaliza con la derrota del fascismo, sino que será una marca que caracterizará su vida. A partir de 1953 en el Uruguay, continúa promoviendo actividades culturales en idioma alemán, se integra a la radioemisora de Gebhardt, funda una compañía teatral y se destaca como escritor y dramaturgo.

El progresivo deterioro de las normas democráticas en el Uruguay, que derivará en la dictadura militar iniciada en 1973, hace que Kalmar, en ese entonces cónsul honorario, desempeñe un importante papel para facilitar el exilio de los perseguidos políticos. El flujo migratorio se invierte y los perseguidos políticos uruguayos encuentran asilo en Austria. Por su actividad como escritor y por su lucha política 
en la resistencia, obtuvo en 2002 el premio Theodor-Kramer, compartido con el anteriormente nombrado Alfredo Bauer.

\section{Consideraciones finales}

Como lo ha señalado el historiador Wolfgang Benz (2001: 8), el estudio del exilio es gran parte estudio de biografías, ya que se pregunta por el destino de individuos, y sólo en la suma de éstos es que se consigue revelar el destino colectivo de la emigración. Estos tres personajes, que no gozaban de un reconocido renombre en Europa, consiguieron, tras la traumática experiencia del exilio, destacarse por su compromiso cultural y político en las sociedades de emigrados. Ellos, artistas e intelectuales de las letras alemanas, se mantienen en los marcos de la misma, y, a pesar del nazismo, no se planteó entre éstos una confrontación de su identidad judeo-alemana, como si ocurrió con tantos otros cercanos a las comunidades judías. Éstos se sentían como artistas e intelectuales pertenecientes a una Alemania democrática que pretendía erradicar los valores del nuevo Reich. Eran opositores al nacionalsocialismo, no sólo por su pertenencia a la religión judía, sino por una contraria concepción política. Eran luchadores políticos, y en ello el judaísmo desempeñó un papel secundario.

Del mismo modo es posible sostener que independiente de la postura política y la identificación religiosa, existe en la amplia mayoría de emigrados en estos países el mantenimiento de un lazo afectivo con la lengua y la cultura alemanas. Eso se explica en parte por el grado de asimilación de los judíos alemanes y austríacos hasta el ascenso del régimen nacionalsocialista, sin distinción entre liberales, sionistas o independientes. "Los judíos [...] se sentían arraigados en la nación alemana, se sentían alemanes, y que alguien los pudiera hacer "extraños' en su propia patria, era algo imposible de ser imaginado". 16

El éxito de las actividades propiciadas por estos exiliados estaba determinado porque el idioma alemán era un elemento importante para la identidad de muchos. El teatro representaba el nexo con la vida cultural perdida, con el mundo lingüístico que se les había arrebatado,

16 Herzig (2005: 240): "Zu stark war [...] das Bewußtsein [der meisten Juden] der tiefen Verwurzelung in der deutschen Gesellschaft. Sie fühlten sich als Deutsche. $\mathrm{Da} ß$ irgend jemand sie zu 'Fremden' in ihrem eigenen Land machen könne, lag außerhalb des Vorstellungsvermögens". 
constituyéndose la patria de la lengua alemana, a pesar de las diferencias, el lugar común.

Igualmente aquellos para quienes las relaciones con su antigua patria no se mantuvieron armoniosas, sino que supusieron una negación de un vínculo identitario con Alemania, como por ejemplo los integrados a las instituciones judías, no renunciaron al idioma, como tampoco a la cultura alemana. No fueron censuradas las actuaciones culturales en el ámbito del teatro, la música, el periodismo, sino aquellas actividades que se vincularan con las agrupaciones políticas antifascistas. Por otra parte, el idioma de comunicación de dichas críticas siguió siendo el alemán, y fueron publicadas en la prensa judía de lengua alemana.

Jacob, Gebhardt y Kalmar fueron artistas comprometidos en la defensa de la cultura alemana y denuncia de la política nazi. En un momento en que parecía ilusorio hablar de una Alemania no nacionalsocialista, estaban convencidos de que la cultura alemana era un bien no renunciable, por ello promovieron la distinción de la misma y de su antigua patria del nombre de Hitler. Como lo expresara Gebhardt:

Nuestro pasado, nuestro presente, nuestro futuro, en este momento nos indican un claro camino: en la lucha de las ideas libres contra el fascismo estamos con cada fibra de nuestro ser junto a los luchadores por la libertad. $^{17}$

\section{Bibliografía}

Arendt, Hannah (2007): Ich will verstehen. München/Zürich: Piper.

Bauer, Alfredo (1995): Zwei Theaterstücke und ein Essay. St. Ingbert: Röhring.

Benz, Wolfgang (2001): Flucht aus Deutschland. München: Deutscher Taschenbuch Verlag.

Benz, Wolfgang/Graml, Hermann/Weiß, Hermann (eds.) (2007): Enzyklopädie des Nationalsozialismus. München: Klett-Cotta.

Blaschitz, Edith (1995): "Bolivia". En: Douer, Alisa/Seeber, Ursula (eds.): Wie weit ist Wien. Lateinamerika als Exil für österreichische Schriftsteller und Künstler. Wien: Picus, pp. 73-77.

17 Carta abierta de Hermann P. Gebhardt a Karl Berets. 14 de diciembre de 1942, PWJ, VII c) 408: "Unsere Vergangenheit, unsere Gegenwart, unsere Zukunft, weist im Augenblick den klaren Weg: Im Kampfe der freiheitlichen Ideen gegen den Faschismus sind wir mit jeder Faser unseres Wesens bei den Kämpfern für die Freiheit". 
Bolbecher, Siglinde/Kaiser, Konstantin (eds.) (2000): Lexikon der österreichischen Exilliteratur. Wien: Deuticke.

Cohen, Robin (1997): "Diasporas, the Nation-State, and Globalisation". En: Gungwu, Wang (ed.): Global History and Migration. Boulder: Westview Press, pp. 117143.

Facal Santiago, Silvia (2006): Auf Wiedersehen Deutschland, Shalom Uruguay. Montevideo: FCU.

Herzig, Arno (2005): Jüdische Geschichte in Deutschland. Bonn: bpb.

Kießling, Wolfgang (1984): Exil in Lateinamerika. Leipzig: Philipp Reclam.

Kloyber, Christian (ed.) (2002): Exilio y cultura. El exilio cultural austríaco en México. México, D.F.: Secretaria de Relaciones Exteriores.

Kohut, Karl/Zur Mühlen, Patrik von (eds.) (1994): Alternative Lateinamerika. Frankfurt am Main: Vervuert/Madrid: Iberoamericana.

Krohn, Claus-Dieter/Zur Mühlen, Patrik von/Paul, Gerhard/Winkler, Lutz (eds.) (1998): Handbuch der deutschsprachigen Emigration 1933-1945. Darmstadt: Primus.

Lemmer, Anne (1999): Freie Deutsche Bühne in Buenos Aires. 1940-1965. Hamburg: Hamburger Arbeitsstelle für deutsche Exilliteratur.

Naumann, Uwe (1985): Ein Theatermann im Exil: P. Walter Jacob. Hamburg: Ernst Kabel.

Pohle, Fritz (1987a): "Paul Walter Jacob am Rio de la Plata. Parte I". En: Exil, 7, 1, pp. 34-52.

- (1987b): "Paul Walter Jacob am Rio de la Plata. Parte II". En: Exil, 7, 2, pp. 3458.

- (1988): "Paul Walter Jacob am Rio de la Plata. Parte III". En: Exil, 8, 1, pp. 7996.

Saint Sauveur-Henn, Anne (ed.) (1998): Zweimal verjagt. Die deutschsprachige Emigration und der Fluchtweg Frankreich - Lateinamerika 1933-1945. Berlin: Metropol.

Spitzer, Leo (2003): Hotel Bolivia. Wien: Picus.

Trapp, Frithjof (1991): “Zwischen Unterhaltungsfunktion und der Erwartung politischer Stellungnahme - Spielplan und künstlerische Konzeption der 'Freien Deutschen Bühne' Buenos Aires”. En: Koch, Edita/Trapp, Frithjof (eds.): Exiltheater und Exildramatik 1933-1945. Maintal: Koch, pp. 118-137.

Trapp, Frithjof/Mittenzwei, Werner/Rischbieter, Henning/Schneider, Hansjörg (eds.) (1999): Handbuch des deutschsprachigen Exiltheaters 1933-1945. München: K. G. Saur.

Wächter, Hans Christof (1973): Theater im Exil. München: Hanser.

Wegner, Sonja (1994): Deutschsprachiges Exil in Uruguay 1933-1945. Tesis de maestría. Essen: Universidad de Essen.

Zur Mühlen, Patrik von (1989): "Politisches Engagement und jüdische Identität im lateinamerikanischen Exil”. En: Schrader, Achim/Rengstorf, Karl Heinrich (eds.): Europäische Juden in Lateinamerika. St. Ingbert: Röhrig, pp. 242-249. 
